\title{
Development of a scale to measure awareness of one's needs
}

\begin{abstract}
Much research has addressed the question of what humans need in order to thrive, with recent research focusing on the fulfillment of basic psychological needs. However, much less attention has been given to the question of awareness of one's needs-that is, identifying what one needs at a particular time and the extent to which one's needs are being met. In principle, greater needs awareness could lead to the fulfillment of those needs. The present study designed and administered a scale to assess needs awareness in a series of studies. The first study examined the factor structure of the new scale, and determined which items best assessed the proposed construct. Additional administrations of the revised scale confirmed the internal consistency of the Needs Awareness Scale, demonstrated its strong test-retest reliability, and established the validity of the measure based on positive correlations with mindfulness and need fulfillment, and negative correlations with neuroticism, anxious attachment style, worry, fear of missing out, and social anxiety. These findings open the door for further avenues of inquiry, including the developmental antecedents of needs awareness, the causal role of this factor in affecting other variables, as well as ways to increase a person's needs awareness.
\end{abstract}

Keywords: needs, mindfulness, motivation, neuroticism, self-determination, adult attachment
Volume 9 Issue 2 - 2018

\author{
Seth J Gillihan,' Emily F Ferguson ${ }^{2}$ \\ 'Haverford College, USA \\ ${ }^{2}$ Center for Autism Research, Children's Hospital of Philadelphia,
} USA

Correspondence: Seth J Gillihan, USA, 355 Lancaster Avenue, Building E2, Haverford, PA 1904I, Tel 610-649-3265, Fax 610537-5059, Email mail@sethgillihan.com

Received: April 08, 2018 | Published: April 24, 2018

\section{Development of a scale to measure awareness of one's needs}

A long line of research has addressed the fundamental question of what humans need in order to thrive. ${ }^{1,2}$ Recent research in this area has focused primarily on psychological need fulfillment. According to Deci \& Ryan's ${ }^{3}$ self-determination theory, the basic psychological needs are relatedness (meaningful connection to others), autonomy (power to make one's own choices), and competence (a sense of being capable and effective). Hundreds of studies have established that fulfillment of these three needs predicts a wide range of positive outcomes, including general life satisfaction, ${ }^{4,5}$ happiness, ${ }^{6}$ mental health, ${ }^{7}$ exercise and dieting behaviors, ${ }^{8}$ identify formation, ${ }^{9}$ and relationship quality. ${ }^{10,11}$ Much less attention has been given to the issue of "needs awareness"- that is, a felt awareness of what one's needs are and the extent to which they are (or are not) being met. It is intuitively plausible to suggest that greater needs awareness will lead to greater need fulfillment; after all, knowing what one's current needs are can lead one to act in ways that fulfill those needs. Murray (1938) defined a need as "the inner feeling of tension which seems to impel us to strive for a certain goal" (p. 64), and posited that "a need ... is inwardly felt as the force of desire" (p. 64). While it may not be necessary for a need to reach conscious awareness in order for one to act on it - we may not realize how tired we were until we get in bed, for example - it is likely that a greater awareness of one's needs will increase the likelihood of meeting those needs. Moreover, existing data suggest that needs awareness may partially determine need fulfillment; for example, individuals who are more self-aware have higher scores on measures of need fulfillment. ${ }^{12}$ However, we are not aware of any studies that directly measure needs awareness (rather than general "self-awareness") or examine associations between needs awareness and other outcomes.
The first step in studying a defined construct is to develop a psychometrically sound measure of it. In the current report we first describe the development of a scale to assess individuals' awareness of their needs, and the scale's psychometric properties among the samples in which we administered it. We then present data on the associations between scores on this scale and participants' scores on related constructs including need fulfillment, mindfulness, neuroticism, attachment style, worry, social anxiety, and fear of missing out on social activities. We end by suggesting directions for future research in this area.

\section{Study I: development of the needs awareness scale}

\section{Study Ia: Item Creation and Scale Refinement}

\section{Method}

Item creation: Scale development began with identifying possible items that address one's needs awareness. Items were generated for various kinds of needs, including general statements about needs awareness (e.g., "I know what activities make me feel content"; "I know what makes me happy"), physical needs like hunger (e.g., "I need to eat and don't realize it" [reverse scored]), as well as psychological needs such as relatedness (Deci \& Ryan, ${ }^{3}$ e.g., "I know what I need from my friends/close relationships"). We developed 21 items for the initial administration.

Participants and procedure: All studies in this report were approved by the institutional review board of the host college. We recruited 404 participants through Amazon.com's Mechanical Turk (MTurk), an online system that allows individuals to participate in research studies and to receive compensation for their time $(\$ 0.30$ in the 
current study). Our sample was relatively evenly split between male $(55.0 \%)$ and female $(44.8 \%)$, with a mean age of $31.6 \mathrm{yr}(S D=11.4$, range $=18-73 \mathrm{yr})$. The sample identified primarily as White $(74.3 \%)$ and African American (9.2\%), and non-Hispanic/Latino (90.3\%). The vast majority of the sample had a high school diploma or equivalent (97.5\%), with $37.9 \%$ completing a bachelor's degree and $10.4 \%$ having completed a graduate degree.

Data analysis: Data were subjected to a categorical exploratory factor analysis (CEFA); the scale was then refined using item response theory (IRT) to identify items that did not contribute meaningfully to scale scores. IRT analyses were conducted using IRTPro (v. 2.1). Global model fit was assessed using RMSEA, item level fit was assessed using the S- $\chi^{2}$ fit statistics, and local item dependence between item pairs was assessed using the standardized LD $\chi^{2}$ fit statistics of Chen $\&$ Thissen. ${ }^{13}$ To determine the best scale, items were removed based on violations of local item dependence, the S- $\chi^{2}$ fit statistics, and low information, with priority given to local dependence and item fit violations. Additionally, marginal reliability was monitored when dropping items. The goal was to eliminate poor fitting items, items with local dependence, and low information while not producing a significant drop in marginal reliability.

\section{Results and discussion}

The CEFA of the full 21 items indicated a 2-factor solution with poor to mediocre fit according to $\mathrm{Hu} \& \mathrm{Bentler}^{14}(1998$; RMSEA $=.085$, $\mathrm{CFI}=.904, \mathrm{TLI}=.881, \mathrm{SRMR}=.058)$. Results from a model that included only the first factor (which comprised 15 of the 21 items) also indicated a mediocre to unacceptable fit (RMSEA=.09). Given the lackluster fit of the 2-factor solution and the relative simplicity of a single factor scale, we opted to use the 15 items from Factor 1 in the IRT analyses. Removal of two items that provided the lowest amount of information resulted in a model with acceptable global fit (RMSEA=.05). All item level fit statistics were not statistically significant according to a $p$-value of .01 , and all item pairs had LD $\chi^{2}$ fit statistics less than 10 . Marginal reliability was .90 for the scale with the remaining 13 items. The test information curve indicated that the final scale does an excellent job measuring needs awareness for participants with a low level of needs awareness to those that are above average on needs awareness; it does not perform as well when participants have higher levels of needs awareness (above +2 standard deviations from the mean). That is, the scale measures needs awareness well for approximately $95 \%$ of the scale's range. Cronbach's alpha for the resulting 13-item scale was .88, indicated strong internal consistency. Consistent with the IRT analysis, removal of any additional items would result in a lower alpha value. Based on the initial study, it appears that our scale does an adequate job of measuring needs awareness, and that the items that survived the IRT analysis tap a single factor. In order to further test the resulting scale, we re-administered it among a new sample to confirm that the items were assessing the same construct.

\section{Study I b: Re-administration of refined scale \\ Method}

Participants: We recruited a new round of participants via MTurk. The sample comprised 403 individuals (46.4\% female) with a mean age of $34.9 \mathrm{yr}(S D=11.6$, range $=18-74 \mathrm{yr})$. Most participants selfidentified as White (73.2\%) and were relatively well educated; $99 \%$ reported having completed at least a high school degree or equivalent, with nearly half (48.5\%) having a bachelor's degree or higher.

\section{Measure}

Needs Awareness Scale (NAS): Awareness of one's needs was assessed using the 13-item scale developed in Study 1a (see Appendix for a complete list of items). Scores were calculated for each participant as the mean of all items.

Data analysis: Participants' scores were analyzed to determine Cronbach's alpha as a measure of internal consistency. We also calculated the means and $S D$ for the scale and for each item.

\section{Results and discussion}

Cronbach's alpha for the scale was .89 , indicating excellent internal consistency and replicating the results from the original administration. The removal of any items would result in a lower alpha value. The scale mean and means for each item are presented in Table 1. The average score for the NAS was $3.75(S D=0.64)$ (Table 1). Findings from the re-administration of the Needs Awareness Scale confirmed that the items assess a single construct, and provided further support for the psychometric soundness of the measure. The next study addressed the scale's test-retest reliability to determine if scores on the measure are consistent over time.

Table I Items, means, and SD for Needs Awareness Scale, Study Ib

\begin{tabular}{lll}
\hline Item & Mean & SD \\
\hline I know what makes me happy. & 3.80 & 0.91 \\
I am aware of what I need to do to feel accomplished. & 3.74 & 1.00 \\
It's easy for me to recognize when I'm tired and need to sleep. & 3.97 & 1.02 \\
If I am sick, I know what I need to do to feel better. & 3.69 & 0.93 \\
If I have many things to do, I am able to decide what I need to do first and what I can do later. & 3.64 & 1.03 \\
If I am unhappy I know why. & 3.36 & 1.11 \\
I know what I need from my friends/close relationships. & 3.56 & 1.02 \\
I recognize when I'm not feeling free to be myself. & 3.85 & 0.88 \\
If I'm feeling unhappy I know what I need to do to feel better. & 3.28 & 1.07 \\
I know how much sleep I need. & 3.8 & 1.06 \\
I know what activities make me feel content. & 4.03 & 0.89 \\
I recognize when I'm feeling pressured. & 4.11 & 0.81 \\
I recognize when I don't have the freedom I need in relationships. & 3.88 & 0.92 \\
Full Scale & 3.75 & 0.64 \\
\hline
\end{tabular}




\section{Study I c:Test-retest reliability}

Two previous administrations of the NAS in separate samples (Studies $1 \mathrm{a}$ and $1 \mathrm{~b}$ ) showed that the scale has strong internal consistency. However, both of these administrations were done at a single point in time; we examined consistency of scores over time by having a new sample of individuals complete the measure at two time points.

\section{Method}

Participants and procedure: We recruited participants again through MTurk; they provided their email addresses at time 1 such that they could be contacted 2 weeks later to complete the scale a second time. Responses were then matched through participants' email addresses. The sample comprised 111 participants with a mean age of $42.3 \mathrm{yr}$ $(S D=14.0$, range $=20-70 \mathrm{yr})$ and was predominantly female $(58.6 \%)$ and White $(75.7 \%)$. More than half of the sample $(56.7 \%)$ had a bachelor's degree or higher. Participants were compensated $\$ 0.25$ at each time point.

Measure and data analysis: We administered the 13-item Needs Awareness Scale developed in Study 1a. Internal validity was determined through calculation of Cronbach's alpha for the scale. Test-retest reliability was assessed through a Pearson's correlation of participant scores on the NAS at times 1 and 2 .

\section{Results and discussion}

Cronbach's alpha values were strong at baseline (.90) and followup (.92). Analyses revealed a strong correlation between scores at times 1 and 2 of Pearson's $r=.83$, indicating good test-retest reliability of the scale. Results from the test-retest analysis suggest that needs awareness is a relatively stable trait. As such, effects of needs awareness on outcomes like need fulfillment are likely to be enduring. Based on the sound psychometric properties demonstrated so far, we administered the NAS among a new sample and also administered questionnaires that we predicted would be associated with needs awareness. These measures would allow us to determine the convergent validity of the measure, based on correlations with well-established constructs. We tested the associations of needs awareness with need fulfillment, attachment style, and neuroticism, fear of missing out, worry, social anxiety, and mindfulness.

\section{Study 2: Identification of needs awareness correlates}

We proposed that an awareness of one's needs increases the likelihood that they will be fulfilled. Thus we hypothesized that needs awareness would be positively correlated with need fulfillment. We also predicted that the NAS would be positively correlated with attachment style, which is defined as the habitual ways a person relates to significant other persons ${ }^{15}$ that develop early in life. ${ }^{16}$ For example, some individuals have a "secure" attachment style in which they feel confident in close relationships and can enjoy emotional intimacy with others, whereas other individuals have a "fearful-avoidant" attachment style and struggle with emotional closeness. ${ }^{17} \mathrm{We}$ hypothesized that a more secure attachment style would be related to greater awareness of one's needs, based on existing research showing that attachment style is related to need fulfillment. ${ }^{18}$ Due to an experimenter error we could examine only anxious attachment style in the present study (see Measures section for more details). The rise in social media use has sparked interest in the fear of missing out (FoMO), which refers to the anxiety individuals may feel from perceiving that others are having rewarding experiences without them. ${ }^{19}$ Awareness of one's needs may be protective against FoMO, given that greater need satisfaction is associated with lower FoMO, ${ }^{19}$ furthermore, greater awareness of one's actual needs may direct attention away from the fear that others are having more fun. Therefore we hypothesized that people who are higher on needs awareness would experience less FoMO. Individuals who lack a clear sense of what they need may be more prone to experience excessive amounts of worry, leading us to predict that NAS scores would be negatively correlated with selfreported worry. The negative correlation between need fulfillment and symptoms of anxiety and depression, ${ }^{20}$ which are related to worry, ${ }^{21,22}$ also suggests that worry and needs awareness will be negatively correlated. We expected social anxiety to be negatively correlated with needs awareness, as well. Social anxiety is positively associated with anxious adult attachment, ${ }^{23}$ and anxious attachment is negatively correlated with need fulfillment. ${ }^{18}$ In light of the expected association between need fulfillment and awareness of needs, we hypothesized that social anxiety symptoms would be negative correlated with scores on the NAS. Measures of need satisfaction are negatively associated with neuroticism, or the tendency to experience negative emotional states. ${ }^{24}$ Accordingly, being aware of one's needs is also likely to lead to behaviors that decrease negative emotions. For these reasons we hypothesized that the NAS would be negatively correlated with a measure of neuroticism. Finally, we hypothesized that NAS scores would be positively associated with trait mindfulness - that is, the tendency to focus one's attention on the present in an open and nonjudgmental way. Greater openness to one's experiences and attention to the present is likely to facilitate greater awareness of what one needs. In line with this hypothesis, previous studies have shown that mindfulness is positively associated with need fulfillment. ${ }^{13}$ To summarize, we hypothesized that scores on the NAS would be positively associated with need fulfillment and trait mindfulness, and negatively associated with anxious attachment, neuroticism, fear of missing out, worry, and social anxiety.

\section{Method}

Participants and procedure: Once again we recruited participants through MTurk, who completed the NAS and additional measures described below. The sample comprised 516 participants with a mean age of $38.2 \mathrm{yr}(S D=13.6$, range=19-70yr) and was predominantly female $(58.2 \%)$ and White $(73.6 \%)$. Approximately half of the sample $(51.9 \%)$ had a bachelor's degree or higher. Participants were compensated $\$ 0.85$ for their time.

\section{Measures}

Needs Awareness Scale (NAS): Awareness of one's needs was assessed using the 13-item scale developed in Study 1. Scores were calculated for each participant as the mean of all items. Internal consistency was strong $(\alpha=.91)$. Basic Need Satisfaction Scale (BNSS): The BNSS ${ }^{25}$ is a 21-item scale that assesses the extent to which individuals' needs for autonomy, competence, and relatedness are met. Respondents indicate the extent to which the item statements describe them, from 1 (not at all true) to 7 (very true). Items assess three domains of need satisfaction: autonomy (e.g., "I generally feel free to express my ideas and opinions"), competence (e.g., "I have been able to learn interesting new skills recently"), and relatedness (e.g., "People in my life care about me"). Higher scores indicate greater need satisfaction. 
Experiences in Close Relationships-Revised (ECR-R): Participants completed the ECR-R scale, ${ }^{26}$ which measures avoidant (e.g., I want to get close to my partner, but I keep pulling back), and anxious (e.g., I need a lot of reassurance that I am loved by my partner) attachment styles. Attachment anxiety and attachment avoidance have been found to correlate significantly with anxiety, depression, interpersonal distress, and loneliness. ${ }^{27}$ As mentioned above we were only able to examine anxious attachment style because we inadvertently omitted items from the second half of the questionnaire, which resulted in our not including questions on avoidant attachment style.

Fear of Missing Out (FoMO): Fear of missing out was measured using a scale developed by Przybylski et al. ${ }^{19}$ Participants were instructed to indicate how true each of 10 statements was of their general experiences on a scale from 1 (not at all true of me) to 5 (extremely true of me). Sample items include: "I get worried when I find out my friends are having fun without me" and "I get anxious when I don't know what my friends are up to." The items showed sound validity and internal consistency $(\alpha=.87) .{ }^{19}$

Penn State Worry Questionnaire (PSWQ): The Penn State Worry Questionnaire ${ }^{2}$ is a 16-item scale that assesses one's tendency toward worry. Respondents rate items on a scale from 1 ("not at all typical of me") to 5 ("very typical of me"), for example, "My worries overwhelm me." The PSWQ has demonstrated demonstrated high internal consistency (e.g., Meyer et al. ${ }^{22} a=.93$ ) and is significantly correlated with trait anxiety $(r=.64)$ as measured by the State-Trait Anxiety Inventory.

Big Five Inventory (BFI), Neuroticism Subscale: The neuroticism subscale of the $\mathrm{BFI}^{28}$ comprises eight items that respondents rateona5point scale from Disagree Strongly to Agree Strongly (e.g., "I see myself as someone who is depressed, blue"). Previous research has established its strong psychometric properties. ${ }^{29}$
Social Phobia Inventory (SPIN): The SPIN consists of 17 items that assess degree of discomfort and avoidance in several social settings (e.g., "Talking to strangers scares me"). Items are rated on a 5-point scale from "Not at all" to "Extremely." The SPIN has demonstrated strong reliability and validity. ${ }^{30}$

Philadelphia Mindfulness Scale (PHLMS): The PHLMS comprises 20 items, half of which assess the present-focused awareness facet of mindfulness (e.g., "When I walk outside, I am aware of smells or how the air feels against my face"); the other 10 items measure the acceptance component of mindfulness (e.g., "I try to distract myself when I feel unpleasant emotions"). Each item is rated from 1 ("Never") to 5 ("Very Often"), with acceptance items reverse scored. Previous research has established the sound psychometric properties of the PHLMS. ${ }^{31}$

Data analysis: We computed participants' scores for each of the eight scales, and then used these scores to determine the Pearson's correlations among the scales. We also analyzed each scale's internal consistency (Cronbach's alpha).

\section{Results}

Results of the analyses supported all hypothesized associations. Scores on the NAS were positively associated with need fulfillment as measured by the BNSS (autonomy, $r=.57$; competence, $r=.61$; relatedness, $r=.54)$ and PHLMS-measured trait mindfulness (awareness, $r=.56$; acceptance, $r=.17$ ), and negatively associated with anxious attachment (ECR-R, $r=-.39$ ), neuroticism (BFI, $r=-.42$ ), fear of missing out (FoMO, $r=-.28$ ), worry (PSWQ, $r=-.33$ ), and social anxiety (SPIN, $r=-.38$ ). All reported correlations were significant atthe $p<.001$ level. See Table 2 for coefficients alpha and additional correlations between Study 2 variables (Table 2).

Table 2 Correlations between needs awareness scale and other measures

\begin{tabular}{|c|c|c|c|c|c|c|c|c|c|c|c|c|}
\hline & & I & 2 & 3 & 4 & 5 & 6 & 7 & 8 & 9 & 10 & II \\
\hline I. & NAS & 0.91 & & & & & & & & & & \\
\hline 2. & BNSS & & & & & & & & & & & \\
\hline 3. & Autonomy & 0.57 & 0.82 & & & & & & & & & \\
\hline 4. & Competence & 0.61 & 0.72 & 0.79 & & & & & & & & \\
\hline 5. & Relatedness & 0.54 & 0.71 & 0.67 & 0.85 & & & & & & & \\
\hline 6. & ECR-R & -0.39 & -0.57 & -0.54 & -0.52 & 0.97 & & & & & & \\
\hline 7. & Neuroticism & -0.42 & -0.57 & -0.59 & -0.51 & 0.56 & 0.9 & & & & & \\
\hline 8. & FoMO & -0.28 & -0.42 & -0.42 & -0.29 & 0.55 & 0.45 & 0.89 & & & & \\
\hline 9. & PSWQ & -0.33 & -0.51 & -0.47 & -0.39 & 0.5 & 0.82 & 0.47 & 0.96 & & & \\
\hline 10. & SPIN & -0.38 & -0.5 & -0.5 & -0.48 & 0.45 & 0.58 & 0.45 & 0.56 & 0.95 & & \\
\hline II. & PHLMS & & & & & & & & & & & \\
\hline 12. & Awareness & 0.56 & 0.27 & 0.31 & 0.32 & 0 & $-.07 p=.11$ & $-.01 \mathrm{p}=.88$ & $.00 p=.99$ & -0.16 & 0.86 & \\
\hline 13. & Acceptance & 0.17 & 0.39 & 0.36 & 0.26 & -0.45 & -0.52 & -0.47 & -0.55 & -0.48 & -0.16 & 0.89 \\
\hline
\end{tabular}

Note. Ns range from 507 to 5 I6. All p-values < .00I unless otherwise designated. Numbers on diagonal represent Cronbach's alpha among this sample. NAS, Needs Awareness Scale; autonomy, competence, and relatedness are subscales of the BNSS (Basic Need Satisfaction Scale); ECR-R, Experiences in Close Relationships-Revised questionnaire; FoMO, Fear of Missing Out scale; PSWQ, Penn State Worry Questionnaire; awareness and acceptance are subscales of the philadelphia mindfulness scale (PHLMS). 


\section{Discussion}

The results from Study 2 confirmed that needs awareness is correlated positively with need fulfillment and mindfulness and negatively with anxious attachment, neuroticism, fear of missing out, worry, and social anxiety. As with any correlational study we cannot know the causal direction of these associations or if a third variable drove the observed correlations. As expected, there were strong associations between needs awareness and all three components of need fulfillment (autonomy, competence, relatedness). These results are consistent with the role of needs awareness in facilitating the fulfillment of our needs. For example, if I know I am starved for human contact, I will be more likely to prioritize getting together with friends. However, other interpretations of this correlation are possible. For example, individuals who tend to have their needs fulfilled may develop a better ability to recognize their own needs. More work needs to be done to explore the important question of how knowing our needs affects having those needs met. We also observed a significant association between needs awareness and multiple measures of negative emotion and anxiety. Several explanations might underlie the association between higher neuroticism and lower needs awareness. First, higher levels of negative emotion might interfere with one's ability to attend to what he or she needs. If so, interventions that lower neuroticism might lead to an increase in needs awareness. Alternatively, not knowing what one needs might contribute to anxiety, depression, and other negative mood states to which individuals with higher neuroticism are especially prone. ${ }^{32}$ Accordingly, increasing one's needs awareness could have a salutary effect on one's level of neuroticism. These explanations are not mutually exclusive, and it may be that neuroticism and low needs awareness are mutually reinforcing. Individuals with an anxious attachment style tended to report lower levels of needs awareness. Anxious attachment is characterized by "a fear of interpersonal rejection or abandonment, an excessive need for approval from others, and distress when one's partner is unavailable or unresponsive". ${ }^{28}$ It may be surprising that individuals with a strong need for approval and connection with others would not score higher on their awareness of needs, since they likely feel their interpersonal needs acutely. However, the excessive nature of the need might make it hard to satisfy, leaving the person with the persistent sense of not knowing what he or she really needs. Not surprisingly, anxious attachment was also negatively correlated with fulfillment of one's need for positive relationships. As expected, awareness of one's needs was positively linked with both the awareness and the acceptance components of mindfulness, which is consistent with research showing that mindfulness predicts need satisfaction (Table 2)..$^{12}$ The correlation between mindful awareness and needs awareness is understandable given the common denominator of awarenessbeing tuned in to one's experience in general predicts being tuned in to one's needs specifically. Interestingly the correlation with needs awareness was greater for mindful awareness $(r=.56)$ than for mindful acceptance ( $r=17$ ), which suggests that knowing what one needs is no guarantee of accepting those needs.

The negative association between needs awareness and FoMO was also consistent with our predictions. One possible explanation for this correlation is that greater awareness of our actual needs protects against the fear that we should have been doing something else that would have been more enjoyable. For example, if John chose to stay in one night instead of going to a party with friends because he needed to get a good night's sleep, his awareness of that need (and perhaps of its importance) could prevent him from dwelling on all the fun he missed when he sees photos of the party on his friends' social media accounts. Greater needs awareness might even predict a lower likelihood of being on social media in the first place, given the positive association between FoMO and social media engagement. ${ }^{19}$ Needs awareness was also negatively associated with three measures of negative emotional or cognitive tendencies: neuroticism, worry, and social anxiety. As with any correlation, more work is needed to determine what drives this association. It may be that greater emotional stability allows for a higher awareness of one's needs, or perhaps greater needs awareness contributes to emotional well-being. The link between needs awareness and social anxiety is consistent with the observed correlation between needs awareness and anxious attachment; accordingly, social anxiety and anxious attachment were positive correlated in the present study.

\section{Overall discussion}

It has been nearly 80 years since Henry Murray ${ }^{2}$ published his seminal work on human needs. The present study builds on work by Murray and many others over the past several decades by defining and measuring a new concept: awareness of one's needs. The data reported here show first that needs awareness is a unitary construct that cuts across the domains assessed by the scale items (e.g., needs for sleep, autonomy, general well-being, relationships). This finding is intriguing given that in principle, awareness of one's needs could vary greatly depending on domain. For example, someone might be highly attuned to physiological needs while being completely out of touch with his or her social needs. The single factor we found in Study 1a does not preclude the likelihood that needs awareness is indeed fractionated for some individuals. Additionally, while our items tapped a broad range of needs, there may be other types of items that would reveal multiple factors under needs awareness. More research is needed to address these questions.

Second, data from the current study demonstrate that needs awareness is relatively stable over time, at least over a span of 2 weeks. This finding further supports the validity of needs awareness as a meaningful and measurable psychological construct, given that reliability is a prerequisite (though not a guarantee) of validity. Questions remain not only about the stability of needs awareness over longer intervals, but also about the development of this capacity. For example, what are the developmental antecedents of needs awareness? By what age does it begin to stabilize as a trait? And perhaps most important, how malleable is needs awareness? Given the link between trait mindfulness and needs awareness, for example, it is possible that training in mindfulness could increase awareness of one's needs. The answer to this and other questions awaits further research. Having established the internal consistency and test-retest reliability of a scale to measure needs awareness, we addressed questions of validity by measuring the association between the Needs Awareness Scale and conceptually linked constructs like need fulfillment and mindfulness. All of the predicted associations were confirmed, suggesting that needs awareness is a meaningful construct that is adequately measured by our scale. At the same time, the magnitude of the correlations (absolute values ranging from $r=.17-.61$ ) suggest the NAS was not simply measuring a construct redundant with these other measures.

\section{Strengths, limitations, and future directions}

The present study introduced and assessed the novel concept of 
needs awareness. Through multiple administrations of a measure we determined the scale's psychometric properties among multiple relatively large samples. The Needs Awareness Scale demonstrated sound reliability and validity, including stability over time and the predicted associations with existing constructs. A limitation of the present study is its reliance entirely on participants' self-report. It cannot be known from these data whether participants are accurate in their assessment of their needs awareness. For example, I might believe and report that I always know what makes me happy, when in reality I am a poor judge in this regard. While this feature is common to selfreport data, it may be particularly important in the future to assess the extent to which NAS scores predict meaningful behavioral outcomes. Creative laboratory-based research studies could address this issue by inducing a measurable need in a participant (e.g., nutritional) and then assessing the participant's awareness of that need. The inability to assess avoidant attachment due to an experimenter error was another limitation of the current study, and one that can be remedied in future studies. It will also be important to extend this line of research to more diverse samples, as the present samples were overwhelmingly White and relatively well educated. Extending this research to youths could also address some of the important developmental questions in this domain - for example, what factors prospectively predict one's needs awareness? Finally, given the apparently beneficial effects of high needs awareness, future research could determine whether and how needs awareness might be increased, and what the downstream effects might be. Perhaps most important, this research could address whether improving one's needs awareness leads to a greater likelihood of meeting those needs.

\section{Conclusion}

The current study developed and refined a measure of awareness of one's needs. The resulting Needs Awareness Scale demonstrated sound reliability and validity, and may be used in subsequent research to address the many outstanding questions in this area. ${ }^{33}$

\section{Acknowledgements}

The authors gratefully acknowledge Jared Harpole, Carol Woods, and Paul Johnson at the Kansas University Center for Research Methods and Data Analysis for their expert assistance in conducting the categorical exploratory factor analysis and item response theory analyses. This work was supported by the Visiting Faculty Research and Travel Fund, Office of the Provost, Haverford.

\section{Conflict of interest}

The authors have no Conflict of interest statement to declare.

\section{Appendix A: Final | 3-Item needs awareness scale}

Please rate the extent to which the following items describe you using the scale below.

Please base your responses on how you actually feel—-there are no right or wrong answers.

\begin{tabular}{lllll}
\hline Not at all true of $\mathbf{m e}$ & Slightly true of $\mathbf{m e}$ & Moderately true of $\mathbf{m e}$ & Very true of $\mathbf{m e}$ & Extremely true of $\mathbf{m e}$ \\
\hline $\mathrm{I}$ & 2 & 3 & 4 & 5 \\
\hline
\end{tabular}

1. I know what makes me happy.

2. I am aware of what I need to do to feel accomplished.

3. It's easy for me to recognize when I'm tired and need sleep.

4. If I am sick, I know what I need to do to feel better.

5. If I have many things to do, I am able to decide what I need to do first and what I can do later.

6. If I am unhappy I know why.

7. I know what I need from my friends/ close relationships.

8. I recognize when I'm not feeling free to be myself.

9. If I'm feeling unhappy I know what I need to do to feel better.

10. I know how much sleep I need.

11. I know what activities make me feel content.

12. I recognize when I'm feeling pressured.

13. I recognize when I don't have the freedom I need in relationships. 


\section{References}

1. Maslow A. Motivation and personality. 3rd ed. New York: Harper and Row; 1987.

2. Murray HA. Explorations in personality: A clinical and experimental study of fifty men of college age. New York: Oxford University Press;1947.

3. Ryan RM, Deci EL. Self-determination theory and the facilitation of intrinsic motivation, social development, and well-being. Am Psychol. 2000;55(1):68-78.

4. Milyavskaya M, Koestner R. Psychological needs, motivation, and well-being: A test of self-determination theory across multiple domains. Personality and Individual Differences. 2011;50:387-391.

5. Tay L, Diener E. Needs and subjective well-being around the world. J Pers Soc Psychol. 2011;101(2):354-365.

6. Sheldon KM, Abad N, Ferguson Y, et al. Persistent pursuit of needsatisfying goals leads to increased happiness: A 6-month experimental longitudinal study. Motiv Emot. 2010;34:39-48.

7. Wei M, Shaffer PA, Young SK, et al. Adult attachment, shame, depression, and loneliness: The mediation role of basic psychological needs satisfaction. Journal of Counseling Psychology. 2005;52(4):591-601.

8. Hagger MS, Chatzisarantis NL, Harris J. The process by which relative autonomous motivation affects intentional behavior: Comparing effects across dieting and exercise behaviors. Motivation and Emotion. 2006;30:307-321.

9. Luyckx K, Vansteenkiste M, Goossens L, et al. Basic need satisfaction and identity formation: Bridging self-determination theory and process-oriented identity research. Journal of Counseling Psychology. 2009;56(2):276-288.

10. Demir M, Özdemir M. Friendship, need satisfaction and happiness. Journal of Happiness Studies. 2010;11(12):243-259.

11. Patrick H, Knee CR, Canevello A. The role of need fulfillment in relationship functioning and well-being: A self-determination theory perspective. J Pers Soc Psychol. 2007;92(3):434-457.

12. Brown KW, Ryan RM. The benefits of being present: Mindfulness and its role in psychological well-being. J Pers Soc Psychol. 2003;84(4):822-848.

13. Chen WH, Thissen D. Local dependence indexes for item pairs using item response theory. Journal of Educational and Behavioral Statistics. 1997;22(3):265-289.

14. Hu LT, Bentler PM. Fit indices in covariance structure modeling: Sensitivity to underparameterized model misspecification. Psychological Methods. 1998;3(4):424-453.

15. Van IJzendoorn MH. Adult attachment representations, parental responsiveness, and infant attachment: A meta-analysis on the predictive validity of the Adult Attachment Interview. Psychological Bulletin. 1995;117(3):387-403.

16. Fraley RC. Attachment stability from infancy to adulthood: Meta-analysis and dynamic modeling of developmental mechanisms. Personality and Social Psychology Review. 2002;6(2):123-151.

17. Leak GK, Cooney RR. Self-determination, attachment styles, and wellbeing in adult romantic relationships. Representative Research in Social Psychology. 2001;25:55-62.
18. La Guardia JG, Ryan RM, Couchman CE, et al. Within-person variation in security of attachment: A self-determination theory perspective on attachment, need fulfillment, and well-being. Journal of Personality and Social Psychology. 2000;79(3):367-384.

19. Przybylski AK, Murayama K, DeHaan CR, et al. Motivational, emotional, and behavioral correlates of fear of missing out. Computers in Human Behavior. 2013;29(4):1841-1848.

20. Ryan RM, Patrick H, Deci EL, et al. Facilitating health behaviour change and its maintenance: Interventions based on self-determination theory. European Health Psychologist. 2008;10:2-5.

21. Borkovec TD, Hu S. The effect of worry on cardiovascular response to phobic imagery. Behav Res Ther. 1990;28(1):69-73.

22. Meyer TJ, Miller ML, Metzger RL, et al. Development and validation of the Penn State Worry Questionnaire. Behav Res Ther. 1990;28(6):487-495.

23. Eng W, Heimberg RG, Hart TA, et al. (2001). Attachment in individuals with social anxiety disorder: The relationship among adult attachment styles, social anxiety, and depression. Emotion. 1(4);365-380.

24. Guay F, Senécal C, Gauthier L, et al. Predicting career indecision: A selfdetermination theory perspective. Journal of Counseling Psychology. 2003;50(2):165-177.

25.Gagné M. The role of autonomy support and autonomy orientation in prosocial behavior engagement. Motivation and Emotion. 2003;27(3):199-223

26. Fraley RC, Waller NG, Brennan KA. An item-response theory analysis of self-report measures of adult attachment. Journal of Personality and Social Psychology. 2000;78(2):350-365.

27. Wei M, Russell DW, Mallinckrodt B, et al. The Experiences in Close Relationship Scale (ECR)-short form: Reliability, validity, and factor structure. Journal of Personality Assessment. 2007;88(2):187-204.

28. John OP, Donahue EM, Kentle RL. The Big Five Inventory-Versions 4a and 54. Berkeley, CA: University of California, Berkeley, Institute of Personality and Social Research; 1991.

29. Benet-Martínez V, John OP. Los Cinco Grandes across cultures and ethnic groups: Multitrait-multimethod analyses of the Big Five in Spanish and English. J Pers Soc Psychol. 1998;75(3):729-750.

30. Connor KM, Davidson JR, Churchill LE, et al. Psychometric properties of the Social Phobia Inventory (SPIN). Br J Psychiatry. 2000;176:379-386.

31. Cardaciotto L, Herbert JD, Forman EM. The assessment of presentmoment awareness and acceptance: The Philadelphia Mindfulness Scale. Assessment. 2008;15(2):204-223.

32. Kendler KS, Kuhn J, Prescott CA. The interrelationship of neuroticism, sex, and stressful life events in the prediction of episodes of major depression. American Journal of Psychiatry. 2004;161(4):631-636.

33. Reiss S, Havercamp SM. Toward a comprehensive assessment of fundamental motivation: Factor structure of the Reiss Profiles. Psychological Assessment. 1998;10(2):97-106. 